REGARDS

SUR L'ECONOMIE ALLEMAND

BULLETIN ECONOMIQUE DU CIRAC
Regards sur l'économie allemande

Bulletin économique du CIRAC

$73 \mid 2005$

Varia

\title{
Une politique sous surveillance
}

Isabelle Bourgeois

\section{OpenEdition}

Journals

Édition électronique

URL : http://journals.openedition.org/rea/202

DOI : 10.4000/rea.202

ISBN : 978-2-8218-0842-3

ISSN : 1965-0787

Éditeur

CIRAC

Édition imprimée

Date de publication : 1 octobre 2005

Pagination : 3-6

ISSN : 1156-8992

Référence électronique

Isabelle Bourgeois, « Une politique sous surveillance », Regards sur l'économie allemande [En ligne], 73 | octobre 2005, mis en ligne le 22 avril 2008, consulté le 15 septembre 2020. URL : http://

journals.openedition.org/rea/202 


\section{Une politique sous surveillance}

En ce début d'automne, la conjoncture allemande évolue sans surprises ; le PIB devrait enregistrer une croissance de $0,8 \%$ en 2005 , comme prévu. La consommation intérieure frémit quelque peu tout en restant très faible. Et comme d'habitude depuis plusieurs années maintenant, ce sont les activités à l'export qui tirent seules l'économie. Leur excellente tenue laisse présager, cette année encore, un excédent commercial record, bien que la flambée des prix pétroliers ait considérablement renchéri les importations et bien que le récent ralentissement de la croissance au sein de la zone euro tasse légèrement la demande de produits allemands. L'industrie voit malgré tout se remplir ses carnets de commande, ce qui contribue positivement au climat des affaires. Dans un contexte mondial qui reste porteur, les entreprises considèrent comme favorables les perspectives sur le long terme.

Leur confiance en l'avenir n'est pas affectée réellement par les incertitudes liées à la constitution du prochain gouvernement fédéral de coalition. Les inquiétudes qu'exprimaient les entreprises durant la campagne électorale sont levées: qui que soit le chancelier, il (ou elle) ne pourra mener qu'une politique de changement dans la continuité. L'inconnue du programme gouvernemental pèse peu en comparaison des effets imprévisibles du cyclone « Katrina » à la fois sur l'évolution des prix pétroliers, de la conjoncture américaine, de la demande mondiale et européenne. Ce sont là des incertitudes autrement réelles sur les perspectives de croissance 2006 d'une économie hautement globalisée.

On s'inquiète à tort, en France, d'une hypothétique crise politique et de ses effets présumés paralysants sur l'évolution de l'économie allemande. Le résultat du scrutin du 18 septembre n'est peut-être pas celui qu'espéraient nombre d'observateurs, mais la République fédérale a toujours un gouvernement et un parlement en mesure de régler les affaires courantes. Et le message des électeurs est on ne peut plus clair : ils ont voté massivement pour la poursuite d'une politique de réformes modérée, ce qui traduit leur volonté de voir leur pays renouer durablement avec la compétitivité, mais pas au prix d'une remise en question du modèle social. Les $8,7 \%$ de voix à mettre à l'actif de la nouvelle gauche marxiste (de même que les $3,9 \%$ attribués à des groupuscules divers, soit moins des $5 \%$ requis pour entrer au Bundestag) révèlent que le vote protestataire reste un phénomène marginal outre-Rhin.

Tous les autres partis ont présenté au cours de la campagne des programmes abondant dans le sens des réformes; chacun bien entendu selon sa perspective idéologique propre. II n'en reste pas moins qu'ils partagent la même analyse que le gouvernement de coalition sortant ou son opposition: seules des réformes structurelles apporteront à terme croissance et emplois. La seule, et réelle, incertitude qui affecte depuis l'été - mais très peu en comparaison de l'évolution des prix pétroliers - le moral des affaires et des ménages concerne dès lors la nature exacte du policy mix que proposera la nouvelle coalition. Mais dès qu'elle sera constituée et que ses orientations politiques générales seront connues, la sérénité s'installera de nouveau.

Car la politique économique et sociale allemande reste dans une large mesure prévisible. Un certain degré de continuité est d'autant plus garanti que, contrairement à ce qu'a pu laisser croire la forte médiatisation d'un affrontement entre SPD/Verts d'un côté, Union chrétienne-démocrate/FDP de l'autre, ce ne sont pas des personnalités isolées qui fixent outre-Rhin le cap politique de la fédération. Celui-ci est défini grâce à un jeu parlementaire complexe associant l'instance collégiale qu'est le gouvernement fédéral, les représentants élus du peuple (groupes parlementaires au Bundestag) et l'exécutif des Länder (via le Bundesrat). Institutionnellement, la politique allemande est toujours une œuvre
Confiance des milieux économiques

Les électeurs ont voté pour une politique de compétitivité

Un nouveau policy mix...

... mais pas de changement de cap 
Au besoin, les organisations professionnelles corrigeront le cap

Ce sont elles qui détiennent la compétence technique

Seule politique de relance possible : les réformes collective. Et, même si elle est le fruit d'ajustements entre les divers programmes électoraux, ses orientations n'en restent pas moins prévisibles dans leurs grandes lignes. C'est cela qui explique pourquoi, bien qu'ils soient déçus du scrutin et des projets d'alliances en discussion, les milieux boursiers et les fédérations professionnelles s'en accommodent. Le tempo des réformes pourrait certes se tempérer, «mais le cap restera inchangé », expliquait par exemple au lendemain des élections Anton Börner, président de la Fédération du Commerce extérieur BGA (dpa, 20-09-05).

Cette confiance en l'avenir des milieux économiques repose aussi sur un mode d'organisation de la société civile qui fait de leurs confédérations (Verbände) des acteurs majeurs dans la définition des orientations de politique économique et sociale. D'une part, elles figurent parmi ces acteurs institutionnels de la 'triade' décisionnelle rassemblant pouvoirs publics, mouvement syndical et patronat, et qui est l'un des pivots de cette forme négociée du capitalisme qu'on appelle le « modèle rhénan ». Sans leur implication active dans le processus de prise de décision, aucune politique durable ou conforme à l'intérêt économique national (social, pour les syndicats) ne peut être menée. Pour avoir voulu mener une politique en contradiction avec ce dogme, le premier gouvernement de coalition SPD/Verts avait dû 'recentrer' sa politique au bout de quelques mois seulement (voir dans ce numéro). Ce modèle de représentation des intérêts organisés contribue à garantir de la sorte un certain équilibre des intérêts qui prémunit l'Allemagne contre toute rupture politique brutale.

D'autre part, alors que le monde politique est soumis généralement au court terme, définissant souvent son action en fonction d'un calendrier électoral particulièrement chargé, puisque les échéances régionales ou communales sont indépendantes de celles de la fédération, les Verbände privilégient le long terme. Défendant depuis leur origine les intérêts des entreprises de leur branche ou secteur (parfois à outrance, mais cela aussi fait partie de leur rôle), ils constituent une sorte de mémoire non seulement des évolutions sectorielles, mais également des mutations macro-économiques, ce qui leur permet d'émettre des propositions fondées sur les orientations politiques des pouvoirs publics. Elles n'excluent pas la critique, loin s'en faut, ni une certaine propension au lamento. II ne faut pas se méprendre dans ce cas : les propos alarmistes diffusés dans l'opinion ont toujours une visée prophylactique. Cela explique par exemple pourquoi, à l'issue du scrutin, les milieux économiques disaient officiellement redouter une grande coalition; c'était pour mieux rappeler aux deux grandes formations qu'il est de leur devoir de se mettre d'accord sur une politique de réformes qui soit moins calibrée sur leurs intérêts partisans parfois contradictoires que définie selon les impératifs de compétitivité de l'Allemagne. Les syndicats ne poursuivent pas une autre stratégie. Tous plaident aujourd'hui pour une « coalition de la raison », comme le formule le patron de la fédération de l'informatique Bitkom, Bernhard Rohleder (Frankfurter Allgemeine Zeitung, 19-09-05).

Car pour une économie allemande qui donne de nets signes de frémissement depuis plusieurs mois, l'ajournement des réformes signifierait le retour à la stagnation. Celles qu'avaient engagées les deux gouvernements Schröder ne peuvent encore produire pleinement leurs effets. Elles sont largement incomplètes (voir REA 65/04), et ne sont que le début d'une politique forcément complexe et de longue haleine visant la compétitivité globale. Le grand mérite du gouvernement de coalition sortant aura été de lancer le processus permettant de rompre l'inertie. C'est sur ce constat que repose aussi la confiance en l'avenir des milieux économiques qui ont vu s'améliorer et s'assouplir le cadre dans lequel ils exercent leurs activités - pas assez encore, mais tout de même. Les pouvoirs publics ont allégé les prélèvements fiscaux, réduit le poids de la bureaucratie et modernisé les marchés financiers. De leur côté, les partenaires sociaux continuent de mener une politique de modération salariale et ont commencé à lever une partie des 'rigidités' du marché de l'emploi en flexibilisant les conditions de travail. En outre, l'intégration croissante du marché communau- 
taire comme de l'espace monétaire créent dans l'environnement européen une stabilité favorable au développement de l'activité.

Enfin, au cours de longues années consacrées à la rationalisation de la production, les entreprises allemandes se sont donné les moyens de conquérir une solide compétitivité, comme en attestent les parts de marché mondiales allemandes, en hausse ininterrompue depuis 2000 , et le classement de l'Allemagne en $3^{\mathrm{e}}$ position mondiale cette année dans le palmarès de compétitivité établi par le World Economic Forum. En 2004, les coûts salariaux unitaires ont baissé pour la première fois depuis sept ans, grâce à une modération salariale continue et à une hausse de $1 \%$ de la productivité horaire. Cette tendance se poursuit depuis : au second trimestre 2005, qui comptait deux jours de plus que la même période 2004, la productivité horaire a augmenté de 0,6 \% en données corrigées de l'effet calendrier. Toujours au second trimestre, la production industrielle (hors BTP) a enregistré une hausse de $+4,5 \%$ en comparaison annuelle, suivie du secteur des transports, du commerce et de l'hôtellerie $(+2,1 \%)$, et des services aux entreprises $(+1,3 \%$; Destatis). Cette bonne tenue a suffi à compenser le recul enregistré par le secteur agricole $(-2,8 \%)$, le BTP $(-1,7 \%)$ et les services publics et privés $(-0,1 \%)$. Au total, au second trimestre 2005 , la croissance du PIB a été de $+1,5 \%$ par rapport au premier, et même de $+1,9 \%$ si on prend pour référence le second trimestre 2004.

En juillet, les entrées en commandes dans l'industrie ont fait un bond de $6 \%$ par rapport à la moyenne des six premiers mois de l'année, confirmant ainsi la tendance à la hausse qu'on observe depuis le début de l'année, explique la Bundesbank (rapport de septembre). Pour l'essentiel, cette hausse est imputable aux commandes étrangères (+11,75\% de juillet à juillet), particulièrement dans le domaine des biens d'investissements qui reste ainsi le moteur par excellence des exportations industrielles allemandes. Les commandes domestiques ont poursuivi en juillet $(+3,25 \%)$ leur légère progression du second trimestre $(+0,75 \%)$. Elles concernent principalement les biens de consommation, ce qui semble confirmer le frémissement de la demande intérieure enregistré dès la fin du printemps.

En effet, la contribution de la demande intérieure à la croissance du second trimestre $(+0,0 \%$ par rapport au trimestre précédent) a été de $+0,3 \%$, assez pour compenser le solde légèrement négatif des échanges extérieurs $(-0,3 \%)$. La tendance du premier trimestre s'est inversée : le solde commercial positif $(+1,2 \%)$ était alors le seul moteur de la croissance $(+0,8 \%)$ face à une demande intérieure en recul $(-0,4 \%)$. Le frémissement de cette dernière au printemps est imputable aux investissements bruts en biens d'équipement $(+0,2 \%)$, aux variations des stocks (avec une contribution de 0,3 point à la croissance) et aux dépenses de consommation publiques (+0,6\%). La consommation privée (ménages et organismes privés à but non lucratif ; elle contribue à $60 \%$ au PIB) a, elle, baissé de $-0,3 \%$ entre le second et le premier trimestre 2005. Autrement dit: les indicateurs conjoncturels domestiques de l'économie allemande restent fragiles et, surtout, contradictoires, bien que s'esquisse un timide regain d'optimisme. Car la comparaison avec le second trimestre 2004 révèle le rôle moteur d'une demande intérieure en hausse de $+1,5 \%$, tirée essentiellement par les investissements en biens d'équipement $(+1,9 \%)$, suivis de la consommation privée $(+0,5 \%)$; quant aux dépenses de consommation publiques, elles ont baissé de $-0,3 \%$. En revanche, le solde commercial $(+0,1 \%)$ n'a guère contribué à la croissance, la hausse des exportations $(+5,4 \%)$ étant absorbée par celle des importations $(+6,2 \%)$, renchéries par un euro fortement apprécié face au dollar et par la flambée des prix pétroliers.

Depuis le cyclone « Katrina », la facture énergétique a encore augmenté, ce qui commence à peser sur l'activité et, bien plus encore sur la consommation des ménages. Leur revenu disponible avait certes augmenté de $+1,7 \%$ au second trimestre (par rapport à 2004), mais cette marge est désormais absorbée par le
Des entreprises à la compétitivité retrouvée

Hausse continue des commandes dans l'industrie

Timide regain de la demande intérieure

Le coût de l'énergie bride la consommation 
Une compétitivité conquise au prix d'une forte inactivité

Priorité à la consolidation budgétaire renchérissement de l'énergie. Destatis a ainsi calculé que les ménages dépenseront cette année 6,8 milliards $€$ de plus pour le carburant, le gaz et le fuel domestique qu'en 2004, soit $176 €$ en moyenne par foyer. Ce sont les dépenses de chauffage qui pèseront le plus lourd dans leur budget, le fuel ayant augmenté de 34,1\% et le gaz de 9,1\%. Or ce sont là les deux principales sources de chauffage des Allemands; et ce poste s'est déjà renchéri de $25 \%$ depuis 1998, gonflant la part de leurs dépenses réservées à l'habitat (un tiers du total ; les loyers sont restés relativement stables). II en va de même du carburant (+28\%) qui représente $14 \%$ de leur budget mensuel. Les précédentes hausses étaient dues à l'écotaxe comme à la flambée des prix pétroliers à l'approche de l'hiver 1999. Elles avaient contribué à amener les ménages à réduire leurs autres dépenses (voir REA 67/04). Ce scénario devrait se reproduire cette année - et contrecarrer le regain d'humeur consumériste dont avaient fait preuve les Allemands en août, à la surprise de l'institut GfK face à des indicateurs de confiance qui avaient retrouvé le niveau de décembre 2001.

Dans ce contexte, l'évolution du marché de l'emploi est sans surprise. Au second trimestre, le nombre d'actifs est passé à 38,9 millions $(+0,1 \%$ en un an ; Destatis). Le nombre des demandeurs d'emploi (critères OIT) était de 4,1 millions $(+1,6 \%)$, ce qui plaçait le taux de chômage à $9,5 \%$. Les données administratives publiées par l'Agence fédérale pour l'emploi font état de 4,65 millions de chômeurs inscrits en septembre 2005, soit un taux de chômage (critères allemands) de 11,2\%. Par rapport à septembre 2004, le nombre de bénéficiaires des divers revenus de substitution a ainsi crû de 393000 personnes, une hausse qui est due pour l'essentiel au changement de régime d'indemnisation introduit par la loi Hartz IV. Mais sur le long terme, la forte inactivité reste la contrepartie de la compétitivité mondiale des entreprises allemandes.

Et cette situation, conjuguée au vieillissement démographique, creuse le déficit. Pour la quatrième année consécutive, l'Allemagne ne respectera pas les critères de Maastricht. Le projet de budget 2006 préparé par Hans Eichel n'y remédie pas non plus, ce qui incite la Commission européenne, maintenant que les élections sont passées, de menacer l'Allemagne d'une procédure de sanction. Par-delà les impératifs de discipline communautaire, cette menace a la même fonction prophylactique que les critiques constructives des fédérations professionnelles allemandes. II s'agit de maintenir la pression sur le gouvernement en cours de constitution pour qu'il s'attelle au dossier de réformes prioritaire entre tous : la consolidation budgétaire.

L'ACTION DU PROCHAIN GOUVERNEMENT fédéral est ainsi placée sous l'étroite surveillance des milieux économiques et sociaux, nationaux et européens. Si la Commission se fait la gardienne de la discipline budgétaire, les Instituts de conjoncture et, surtout, le Conseil des Sages, ne manqueront pas de rappeler à l'ordre le successeur de Gerhard Schröder et de le pousser à la poursuite des incontournables réformes structurelles : fiscalité, financement des systèmes des retraites et d'assurance maladie, réforme de l'administration, libéralisation du marché du travail, baisse des prélèvements. Tous lancent d'ores et déjà des signaux clairs aux milieux politiques : ces réformes sont urgentes, aucune phase d'expérimentation n'est plus permise (contrairement aux débuts des deux mandats de Gerhard Schröder). II en va de l'attractivité du site Allemagne pour les investisseurs. II en va de la compétitivité d'une Union européenne dont la croissance potentielle est elle aussi en baisse. Il en va, enfin, de la compétitivité globale de l'économie allemande que la mollesse persistante de sa croissance a reléguée en $15^{\mathrm{e}}$ position (après deux années en $13^{\mathrm{e}}$ ) dans le palmarès établi par le World Economic Forum, le principal risque pour la dynamique de croissance allemande résidant dans la propension nationale au pessimisme. Raison de plus pour placer un espoir sans illusion dans la politique du nouveau gouvernement.

Isabelle Bourgeois (03-10-2005) 